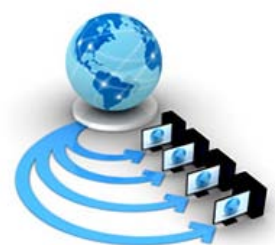

Volume 8, No. 7, July - August 2017

International Journal of Advanced Research in Computer Science

RESEARCH PAPER

Available Online at www.ijarcs.info

\title{
SIMULATIVE INVESTIGATION OF 16X5 GB/S \& 16X10 GB/S WDM-FSO TRANSMISSION SYSTEM UNDER DIFFERENT ATMOSPHERIC CONDITIONS
}

\author{
Jaskaran kaur/ M.tech \\ (Scholar) \\ Department of Electronic \& Communication Engg. \\ SBBS University \\ Punjab, India
}

\author{
Manpreet kaur \\ /Assistant professor \\ Department of Electronic \& Communication Engg. \\ SBBS University \\ Punjab, India
}

\begin{abstract}
In Free Space Optic (FSO) system free space requires as a communication media. Atmospheric attenuation due to communication media can degrade a system performance. To overcome this losses BER or Q-factor of a system are improved by using different type of photodetector at receiver side \& using optical receiver at link.. This paper describe the design and simulation of 16-channel WDM based Free Space Optical communication link under various Atmospheric conditions at different bit rates (5Gbps/10Gbps) using APD \& PIN photo-detector \& the performance of system using APD \& PIN photo-detector is compared. The NRZ modulation format \& semiconductor optical amplifier (SOA) is used in this design. In this simulation it observed that APD photo-detector at bit rate 5Gbps/10Gbpsgive better performance at higher attenuation.
\end{abstract}

Keywords: APD photo-detector, Atmospheric attenuation, Bit Error Rate, PIN photo-detector, Bit analyzer, 16-channel, WDM, Q-factor.

\section{INTRODUCTION}

Free Space Optical Communication system is widely used now a day's due to its high Bit Rate, huge bandwidth and minimum bit error. FSO system provide very efficient communication. In FSO communication system electrical signal first converted into light form for this purpose Laser source is used which include LED's and Laser diodes. On the other hand at receiver side optical-detector is used to covert light signal back into electrical form. [1] The photodetector is an essential element of any optical communication system which connected at receiver side as shown in "Fig.1".

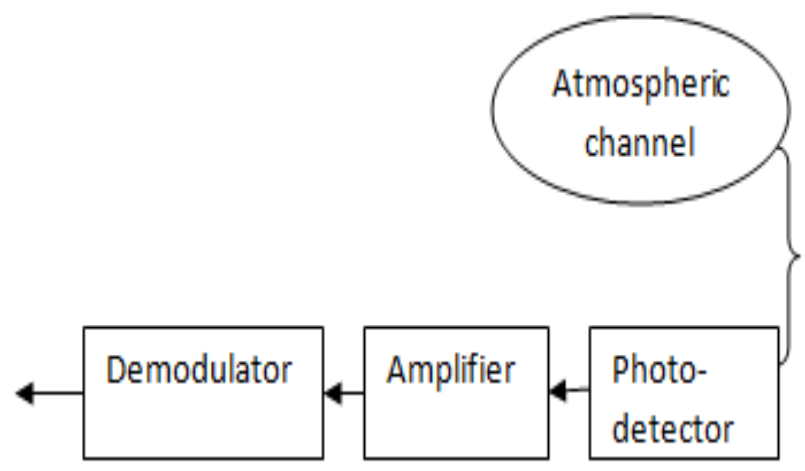

Fig.1. Receiver Section of FSO system

There are two types of photo-detector used in FSO communication system as:
I.
PIN photo-detector
APD photo-detector

II.

PIN photo-detector-PIN photo-detector is mainly used FSO communication system to convert optical signal into electrical form. The PIN photodiode is mainly operates on reverse bias voltage. This was invented in 1950's. A PIN diode mainly included three region as:
a.
b.
$\mathrm{N}$-region
c.
P-region
Intrinsic region

First of all electron-hole pairs are generated in depletion region by photo absorption. These generate more pair of

electron and hole through impact ionization which swept out depletion region very fast i.e the transit time is very less.[2,3]

APD photo-detector-An Avalanche photo-detector (APD) is a semiconductor device which exploits the photoelectric effect to convert light to electricity. APD photo-detector provide a built-in first stage of gain. APD was invented in 1960's. APD structural configuration is same as of PIN photodiode. When high reverse bias voltage is apply to APD it show an internal current gain effect. This internal current gain effect is due to impact ionization. [4] In APD higher gain is possible due to internal multiplication. Where in PIN photo-detector current at output is proportional to light incident. So gain in case of PIN photo-detector is low compared to APD photo-detector.

\section{SYSTEM DESIGN}

The FSO system is sub divided into three parts : Transmitter, FSO channel, Receiver. The Transmitter section has four subsystem: Pseudo Random Bit Stream to generate the sequence of bit. NRZ Modulator to encode the signal. CW array laser for transmission of light signal and forth is Mach-Zehnder modulator to modulate the data.[5] The basic transmitter section is shown in "Fig.2." 


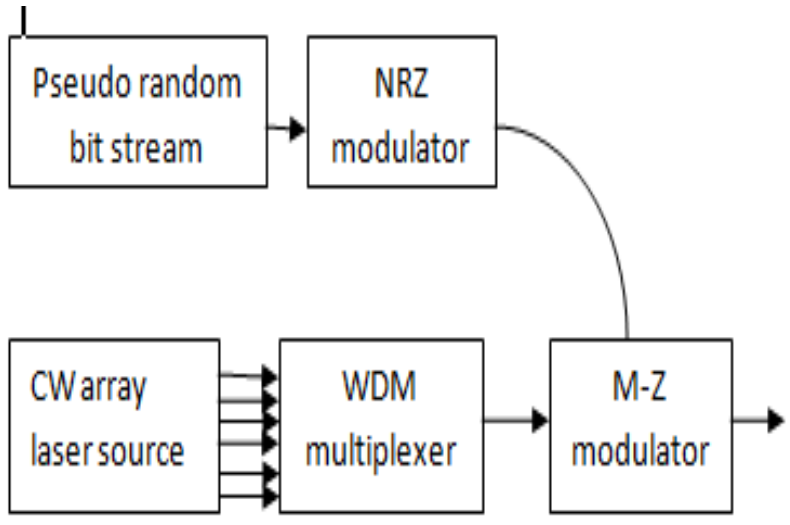

Fig.2. Transmitter section

At the receiver section: Photo-detector is used for conversion of light to electricity. A LPF is used for amplification purpose. 3R Regenerator and BER Analyzer at end of receiver to analyzed the Bit Error Rate and Qfactor.[6] The "Fig.2" show the receiver section of WDMFSO system

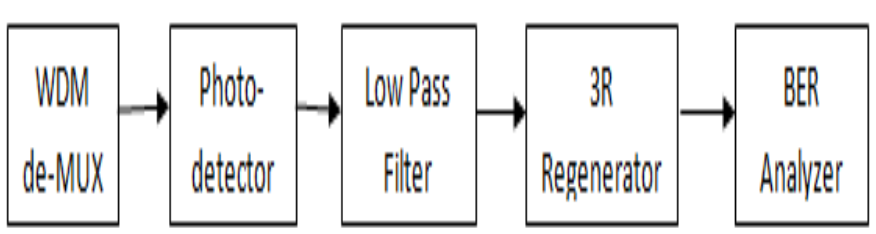

Fig.3. Receiver Section

There are two system design in this paper System I \& System II. Which are discuss as below:

System I. In system I 16x5Gbps system is design using APD \& PIN photo-detector. Firstly 16x5Gbps system using APD photo-detector is investigated for 4 different atmospheric conditions. Then 16x5Gbps system is design using PIN photo-detector.[7]

System II. In this system 16x10Gbps system is design using APD \& PIN photo-detector.16x10Gbps system is design firstly using APD photo-detector. Investigate it for very clear, clear, light haze, heavy haze atmospheric attenuation. Secondly design 16x10Gbps system using PIN photodetector and comparing it with 16x10Gbps system using APD photo-detector.[8] The simulation parameter are shown in Table .I.

Table I. Simulation parameter

\begin{tabular}{|c|c|}
\hline Parameter & Value \\
\hline Bit Rate & 5Gbps, 10Gbps \\
\hline CW array laser frequency & $193.1 \mathrm{THz}-193.16 \mathrm{THz}$ \\
\hline Transmitter aperture diameter & $10 \mathrm{~cm}$ \\
\hline Receiver aperture diameter & $20 \mathrm{~cm}$ \\
\hline Beam divergence & $1 \mathrm{mard}$ \\
\hline
\end{tabular}

\begin{tabular}{|c|c|}
\hline Photodiode gain & 3 \\
\hline Responsivity & $1 \mathrm{~A} / \mathrm{W}$ \\
\hline Dark current & $10 \mathrm{~A}$ \\
\hline
\end{tabular}

\section{RESULT AND DISCISSION}

The 16-channel WDM based Free Space Optical communication link has been analyzed at 5Gbps/10Gbps in term of Bit Error Rate and Q-factor. The Eye diagram for 16X5 WDM-FSO system using APD \& PIN photo-detector are shown in "Fig.4" and "Fig.5" respectively.[9] The parameters Q-factor and BER are mention in Table. II.

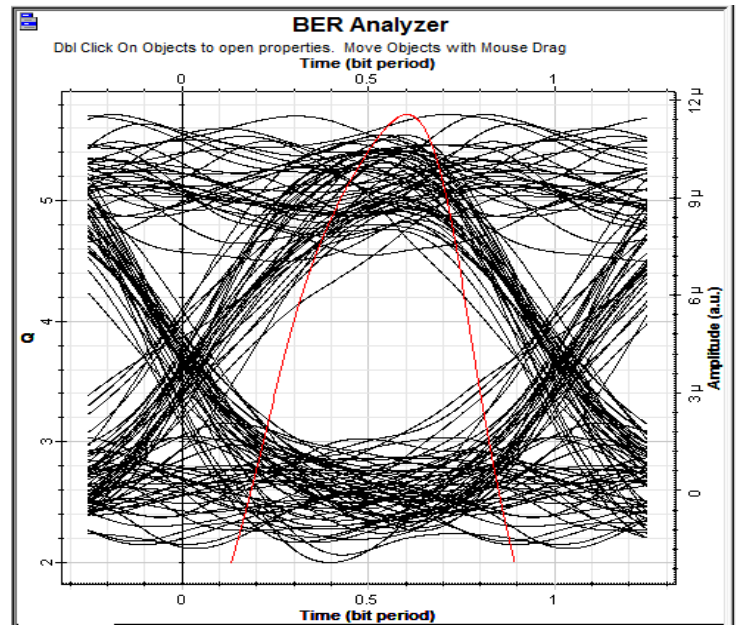

(a)

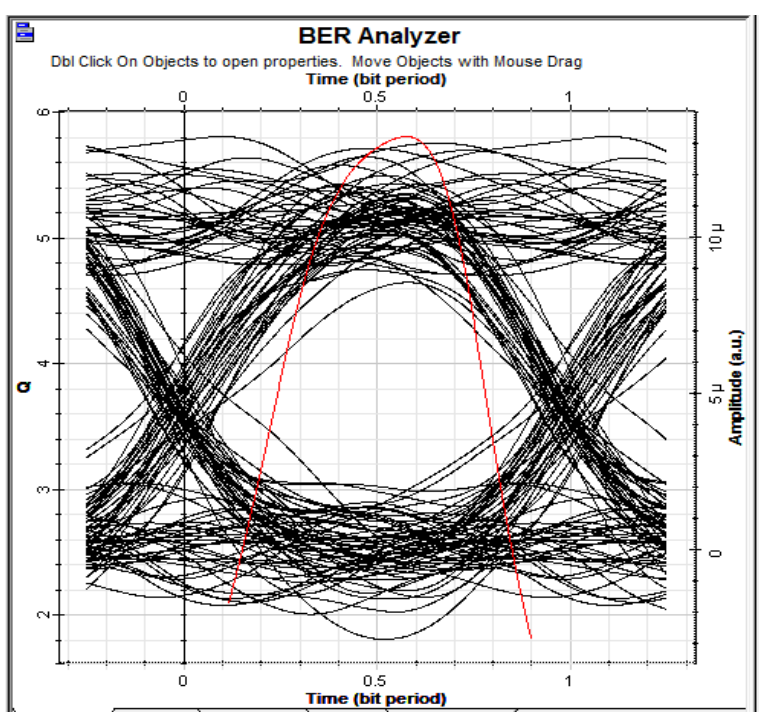

(b) 


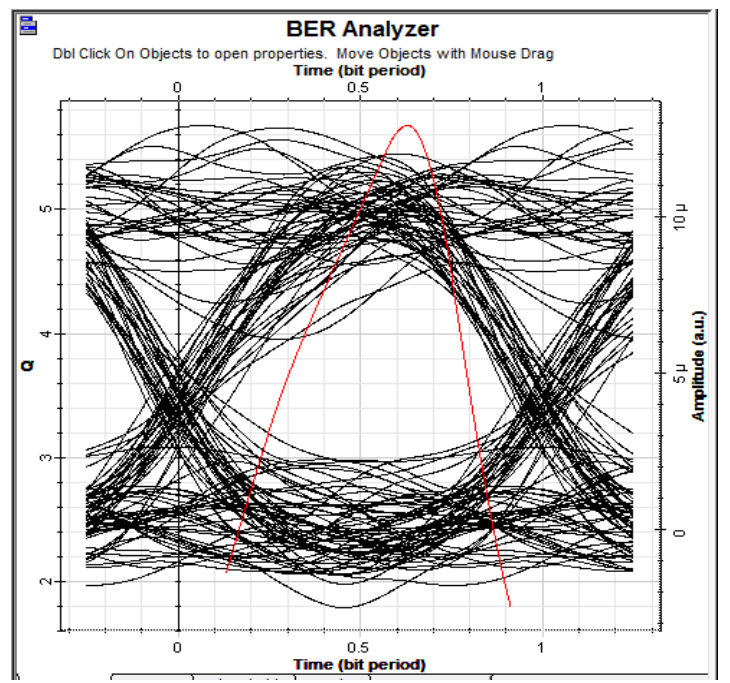

(c)

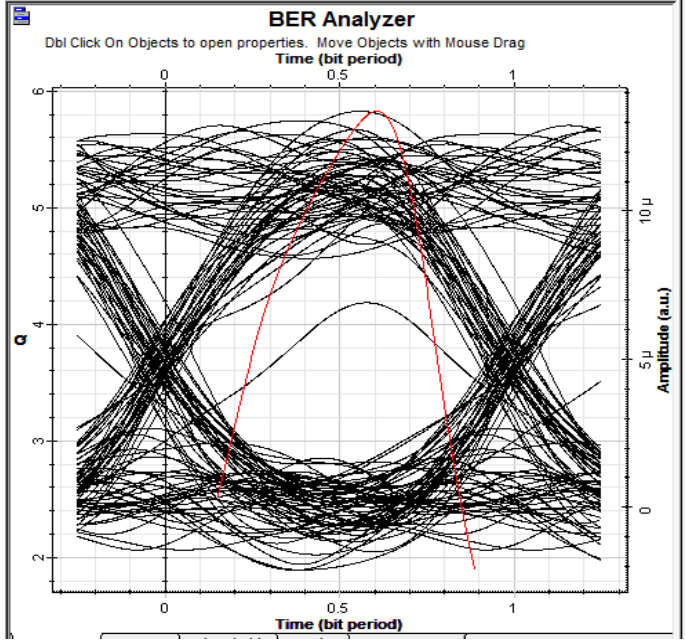

(d)

Fig.4. Eye diagram of 16x5Gbps system using APD photodetector for different atmospheric conditions (a) Very clear sky (b) Clear sky (c) Light haze (d) Heavy haze

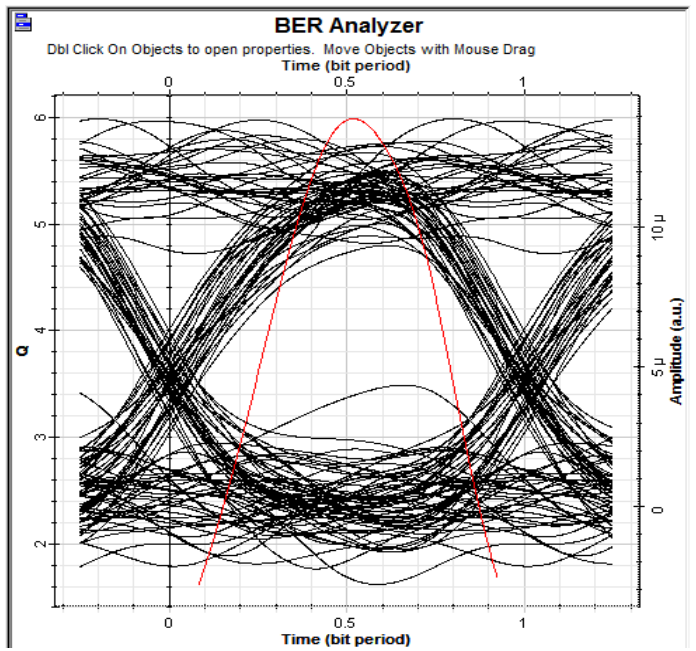

(a)

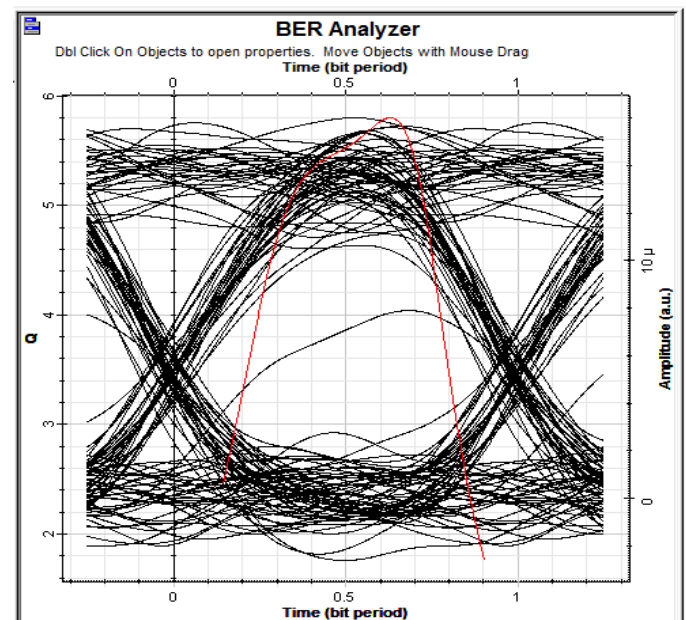

(b)

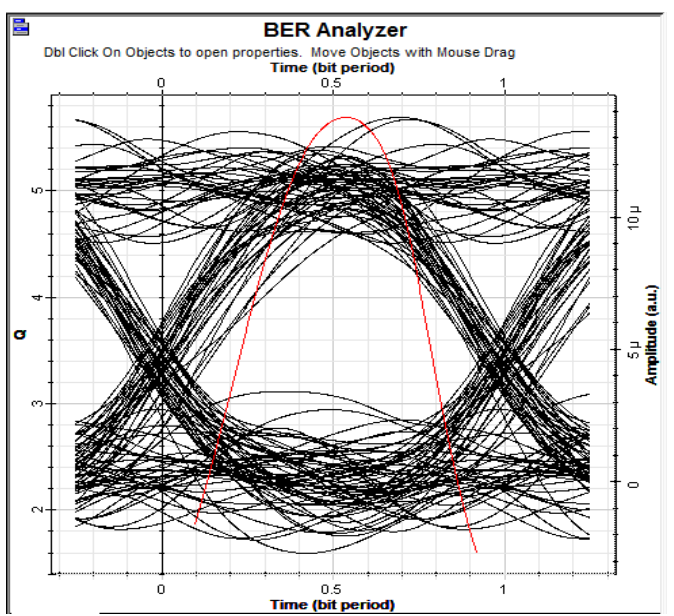

(c)

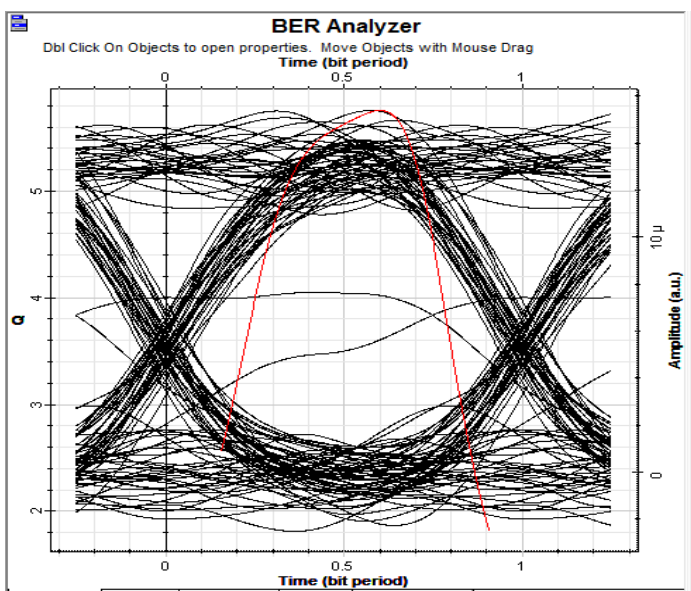

(d)

Fig.5. Eye diagram for 16x5Gbps WDM-FSO system using PIN photo-detector for different atmospheric conditions (a) Very clear sky (b) Clear sky(c) Light haze (d) Heavy haze

Table II. Performance analysis of 16X5Gbps WDM-FSO communication link using APD \& PIN photo-detector for different Atmospheric conditions

\begin{tabular}{|c|c|c|c|c|}
\hline \multicolumn{5}{|c|}{ 16x5Gbps using APD photo-detector } \\
\hline $\begin{array}{c}\text { Atmospheric } \\
\text { Conditions }\end{array}$ & $\begin{array}{c}\text { Very } \\
\text { clear sky }\end{array}$ & $\begin{array}{c}\text { Clear } \\
\text { sky }\end{array}$ & $\begin{array}{c}\text { Light } \\
\text { haze }\end{array}$ & $\begin{array}{c}\text { Heavy } \\
\text { haze }\end{array}$ \\
\hline Attenuation & 0.006 & 0.22 & 0.53 & 2.3 \\
\hline
\end{tabular}




\begin{tabular}{|c|c|c|c|c|}
\hline Power & 3 & 10 & 20 & 32 \\
\hline Link Range & 453 & 198 & 111 & 35 \\
\hline BER & $\begin{array}{c}5.47209 \\
\mathrm{e}^{-009}\end{array}$ & $\begin{array}{c}3.20549 \\
\mathrm{e}^{-009}\end{array}$ & $\begin{array}{c}6.86507 \\
\mathrm{e}^{-009}\end{array}$ & $\begin{array}{c}2.74104 \\
\mathrm{e}^{-009}\end{array}$ \\
\hline Q-factor & 5.71484 & 5.80563 & 5.67672 & 5.82803 \\
\hline \multicolumn{5}{|c|}{ 16x5Gbps using PIN photo-detector } \\
\hline Attenuation & 0.006 & 0.22 & 0.53 & 2.3 \\
\hline Power & 3 & 10 & 20 & 32 \\
\hline Link Range & 388 & 176 & 102 & 32.8 \\
\hline BER & $\begin{array}{c}1.08542 \\
\mathrm{e}^{-009}\end{array}$ & $\begin{array}{c}3.23167 \\
\mathrm{e}^{-009}\end{array}$ & $\begin{array}{c}6.2838 \\
\mathrm{e}^{-009}\end{array}$ & $\begin{array}{c}4.14631 \\
\mathrm{e}^{-009}\end{array}$ \\
\hline Q-factor & $\begin{array}{c}5.98384 \\
5.79955\end{array}$ & 5.69074 & 5.75565 \\
\hline
\end{tabular}

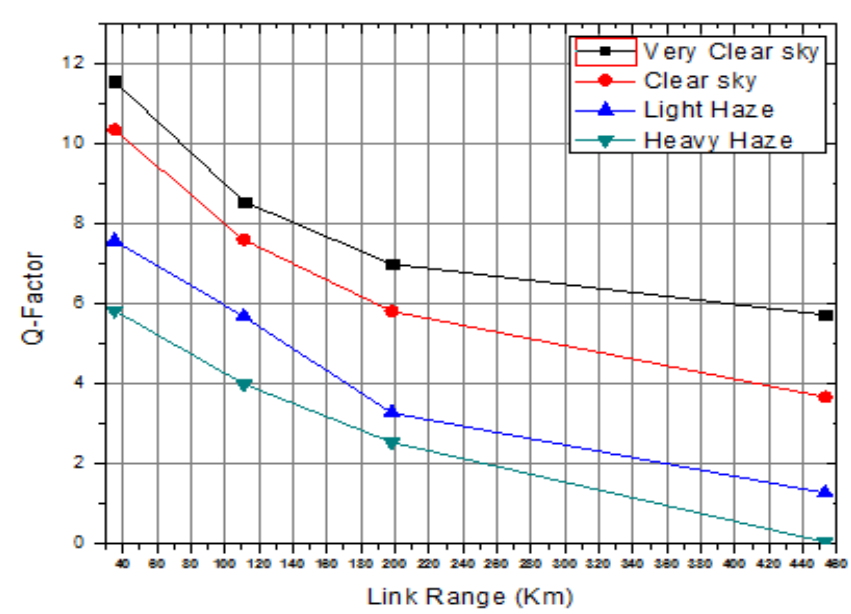

(a)

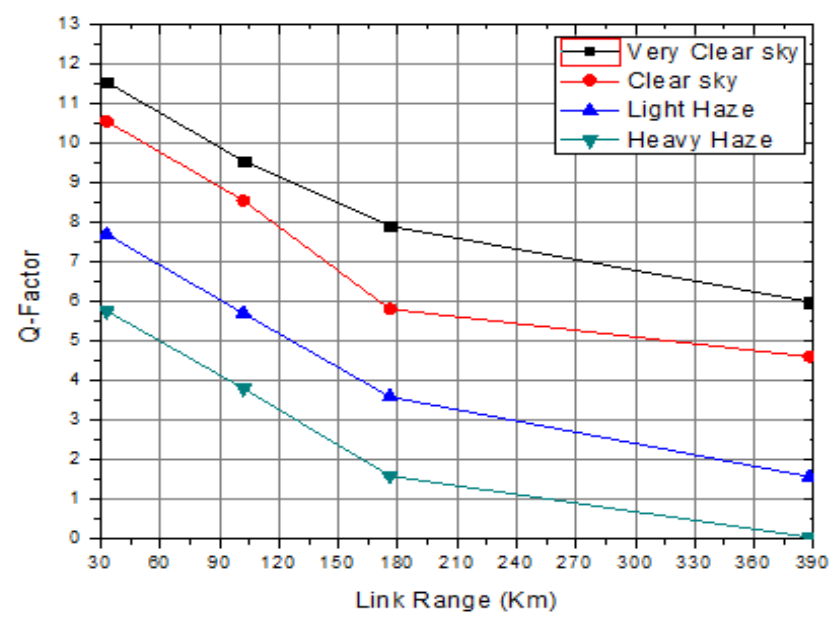

(b)

Fig.6. Q-Factor v/s Link Range for 16x5Gbps WDM-FSO system (a)using APD photo-detector (b) using PIN photo-detector

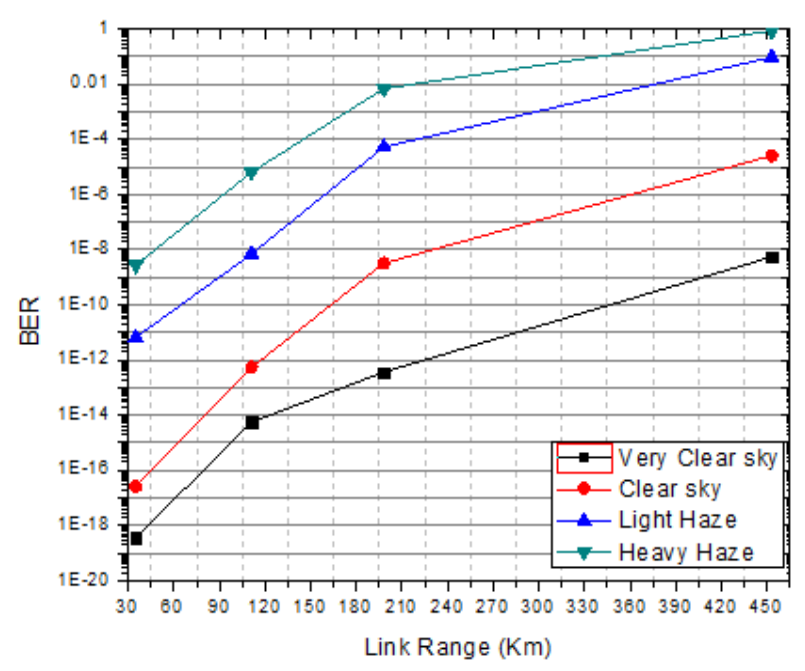

(a)

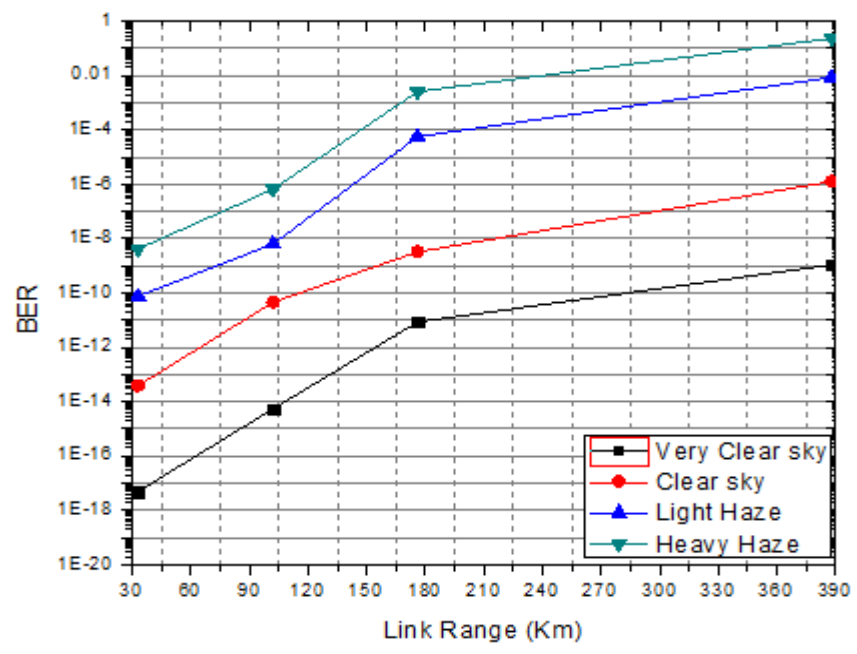

(b)

Fig.7. BER v/s Link Range for 16x5Gbps WDM-FSO system (a)using APD photo-detector (b) using PIN photo-detector

Similar as $16 \mathrm{X} 5 \mathrm{Gbps}$ a $16 \mathrm{X} 10 \mathrm{Gbps}$ system is design using APD \& PIN photo-detector. 16X10Gbps system using APD photo-detector give better result as compare to 16X10 using PIN photo-detector. The Eye diagram foe 16X10Gbps WDM-FSO system using APD\& PIN photo-detector are shown in "Fig.8" and "Fig.9". The parameters Bit Error Rate, Link Range and Quality factor are mention in Table

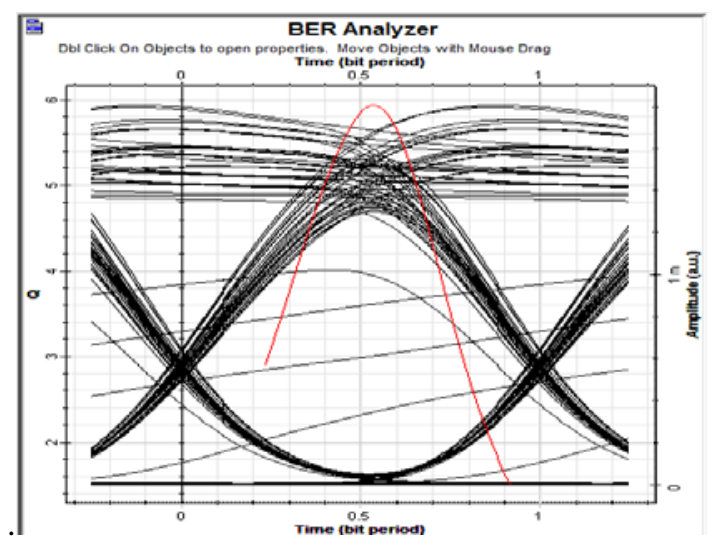

(a) 


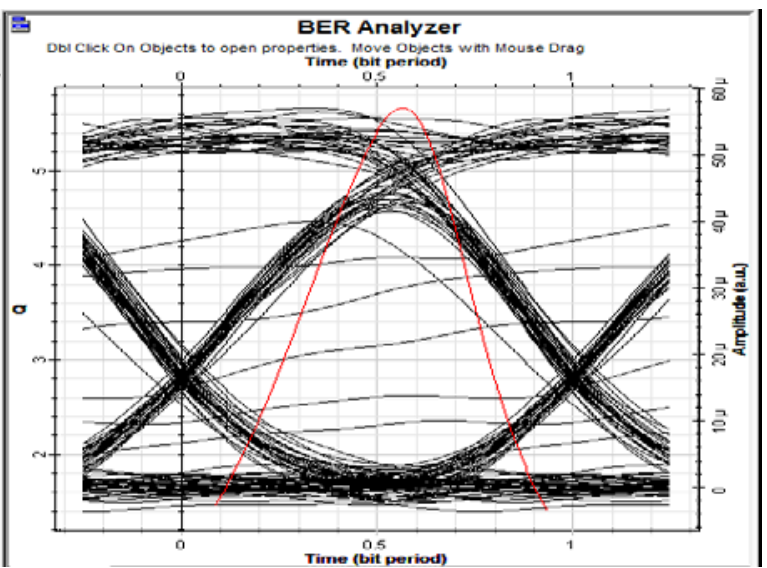

(b)

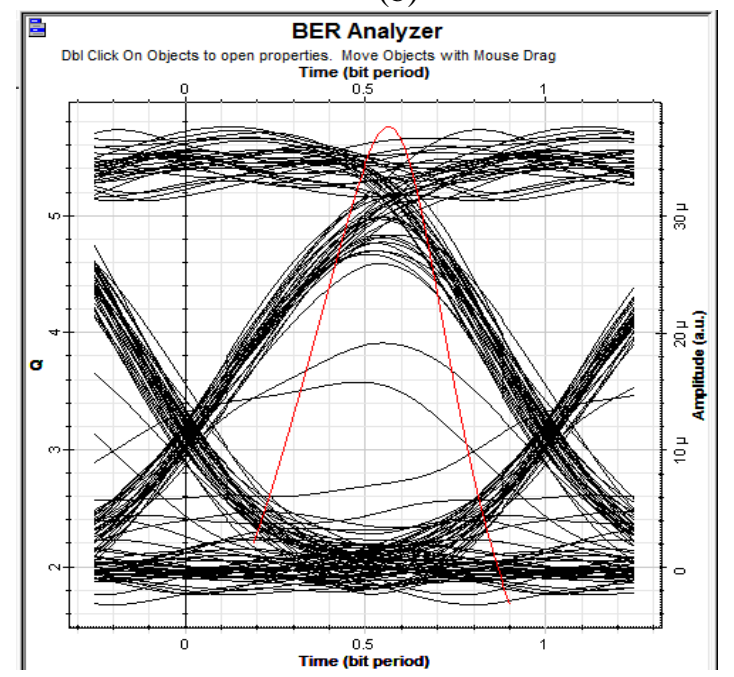

(c)

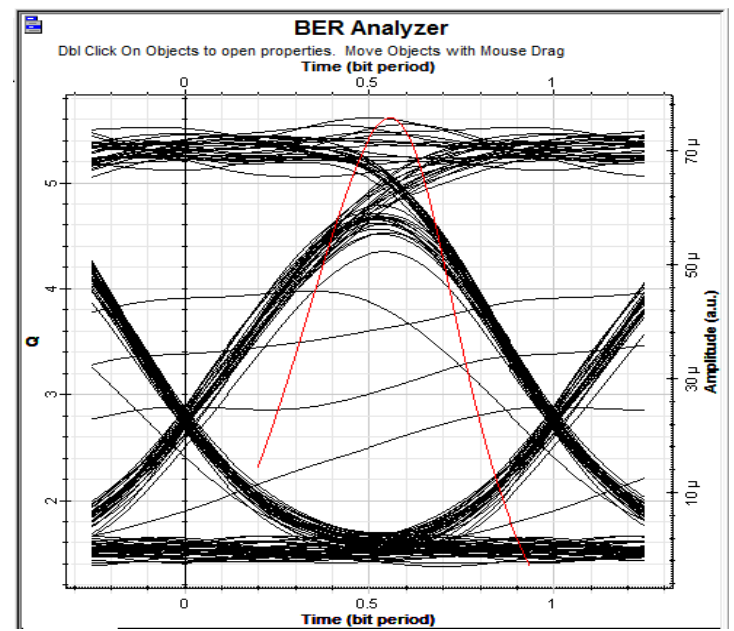

(d)

Fig.8. Eye diagram for 16X10Gbps WDM-FSO system using APD photo-detector for different atmospheric conditions (a) Very clear sky (b) Clear sky (c) Light haze (d) Heavy haze

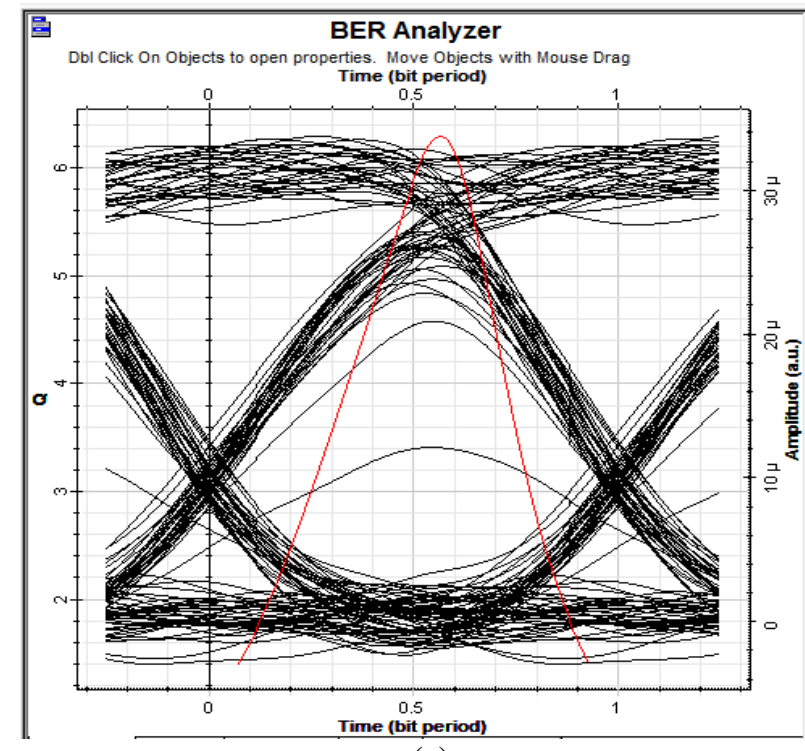

(a)

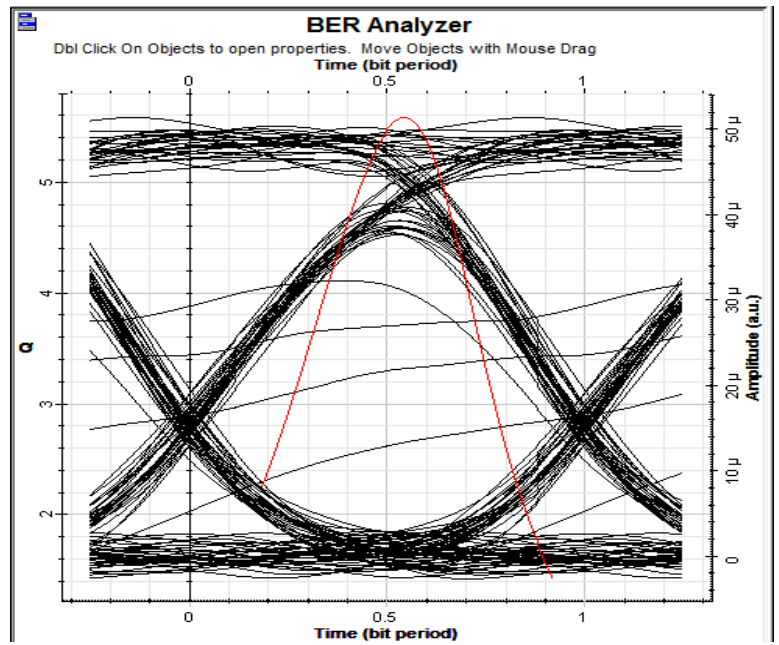

(b)

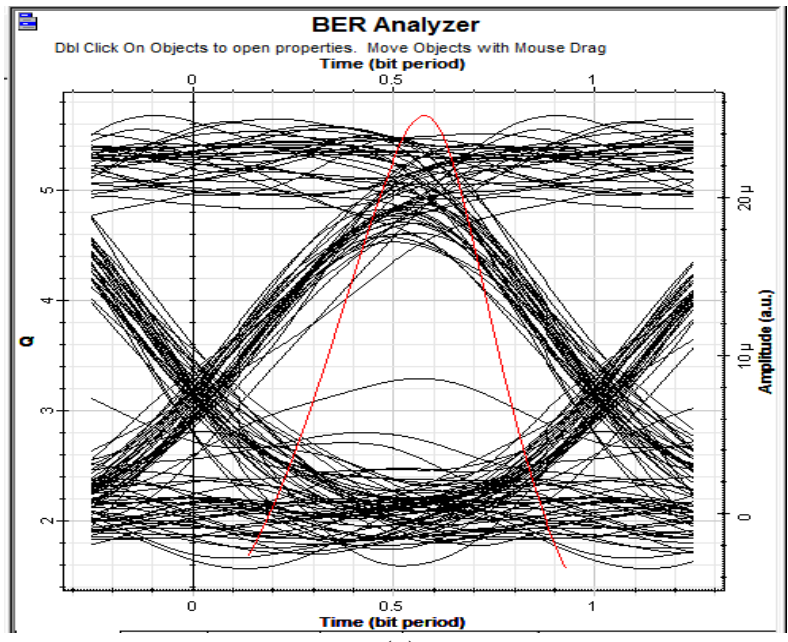

(c) 


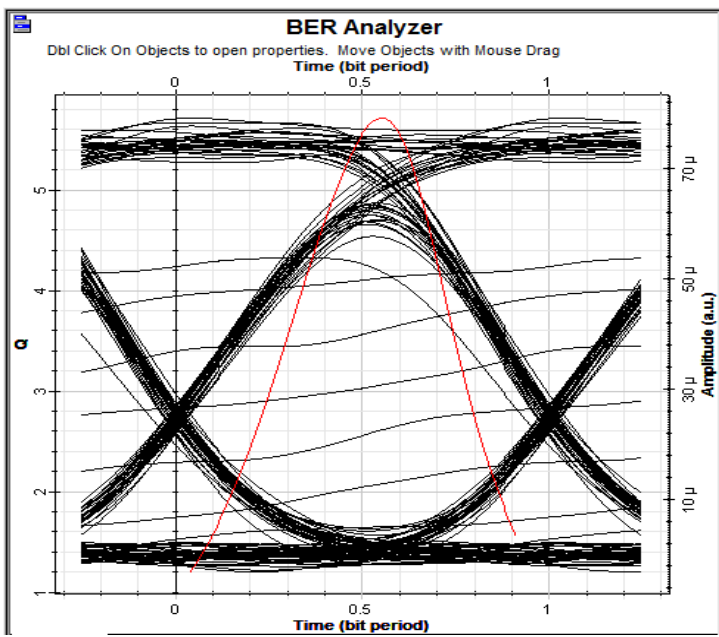

(d)

Fig.9. Eye diagram for 16X10Gbps WDM-FSO system using PIN photo-detector for different atmospheric conditions (a) Very clear sky (b) Clear sky (c) Light haze (d) Heavy haze

Table III. Performance analysis of 16X10Gbps WDM-FSO communication link using APD \& PIN photo-detector for different conditions

\begin{tabular}{|c|c|c|c|c|}
\hline \multicolumn{5}{|c|}{ 16x10Gbps using APD photo-detector } \\
\hline $\begin{array}{l}\text { Atmospheric } \\
\text { conditions }\end{array}$ & $\begin{array}{l}\text { Very } \\
\text { clear sky }\end{array}$ & $\begin{array}{l}\text { Clear } \\
\text { sky }\end{array}$ & $\begin{array}{l}\text { Light } \\
\text { haze }\end{array}$ & $\begin{array}{l}\text { Heavy } \\
\text { haze }\end{array}$ \\
\hline Attenuation & 0.04 & 0.19 & 0.52 & 2.3 \\
\hline Power & 10 & 10 & 20 & 35 \\
\hline Link Range & 370 & 194 & 104 & 33 \\
\hline BER & $\begin{array}{c}8.94084 \\
\mathrm{e}^{-010}\end{array}$ & $\begin{array}{c}6.52781 \\
\mathrm{e}^{-009}\end{array}$ & $\begin{array}{c}4.20817 \\
\mathrm{e}^{-009}\end{array}$ & $\begin{array}{c}7.02098 \\
\mathrm{e}^{-009}\end{array}$ \\
\hline Q-factor & 5.93701 & 5.66413 & 5.75753 & 5.61365 \\
\hline \multicolumn{5}{|c|}{ 16x10Gbps using PIN photo-detector } \\
\hline Attenuation & 0.04 & 0.19 & 0.52 & 22.3 \\
\hline Power & 10 & 10 & 20 & 35 \\
\hline Link range & 590 & 170 & 99 & 31 \\
\hline BER & $\begin{array}{c}1.51539 \\
\mathrm{e}^{-010}\end{array}$ & $\begin{array}{c}9.24711 \\
\mathrm{e}^{-009}\end{array}$ & $\begin{array}{c}6.66409 \\
\mathrm{e}^{-009}\end{array}$ & $\begin{array}{c}4.79512 \\
\mathrm{e}^{-009}\end{array}$ \\
\hline Q-factor & 5.93546 & 6.29388 & 5.5825 & 5.68096 \\
\hline
\end{tabular}

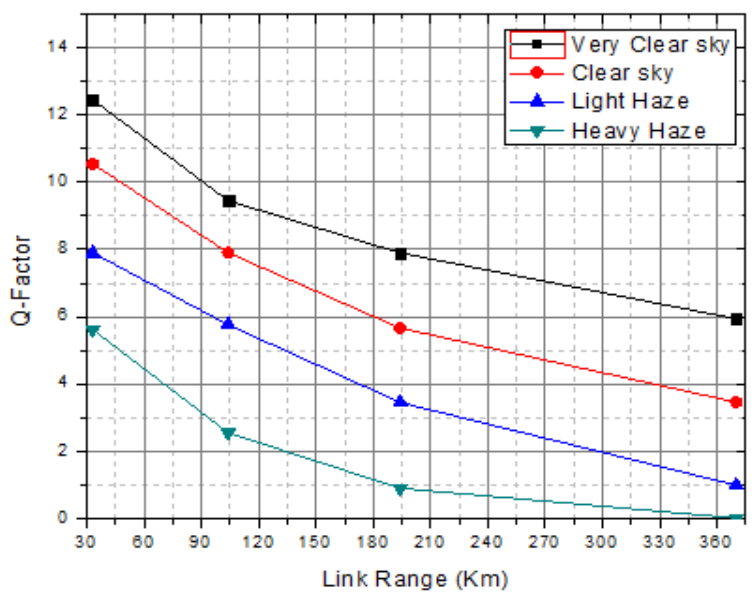

(a)

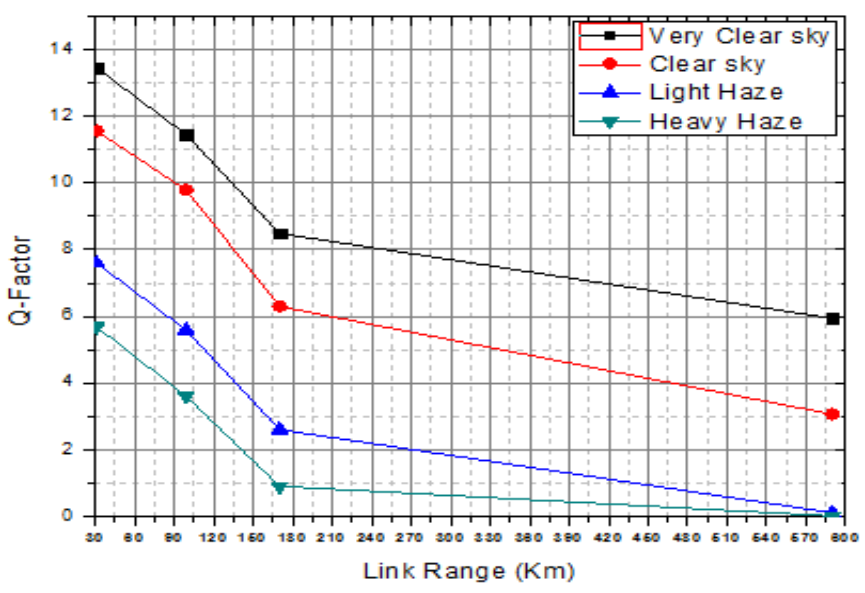

(b)

Fig.10. Q-Factor v/s Link Range for 16x10Gbps WDM-FSO system (a)using APD photo-detector (b) using PIN photo-detector

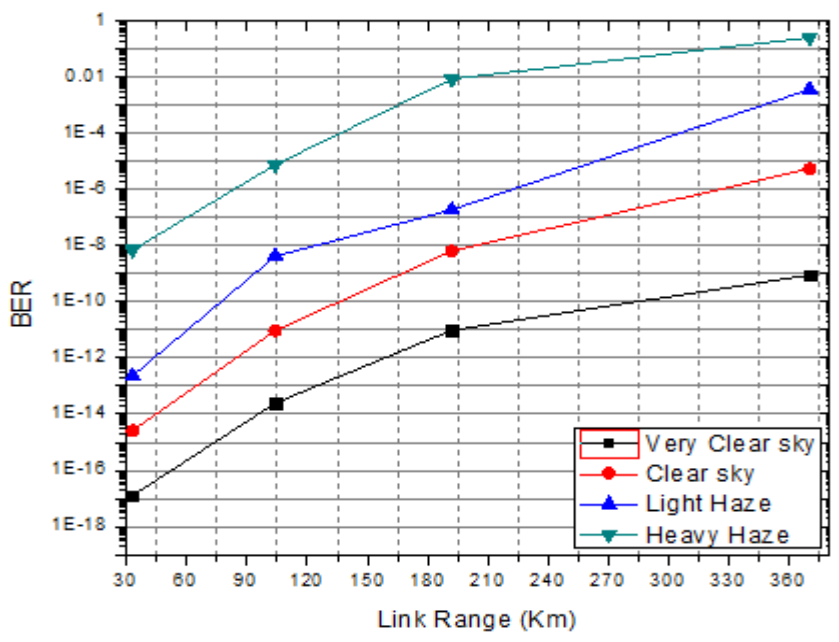

(a)

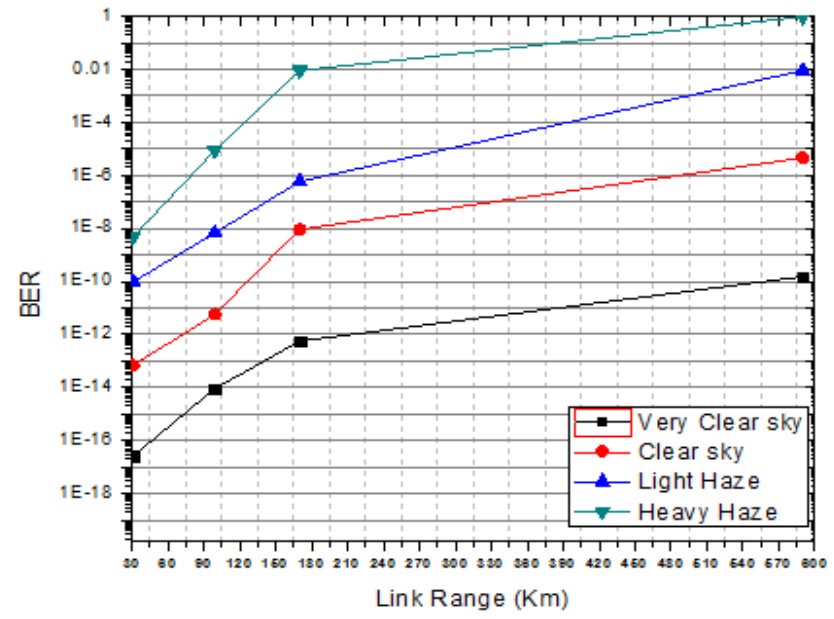

(b)

Fig.11. BER v/s Link Range for 16x10Gbps WDM-FSO system (a)using APD photo-detector (b) using PIN photo-detector

\section{CONCLUSION}

In this paper, 16-channel WDM based Free Space Optical transmission system is design and investigate at 5Gbps/10Gbps with APD \& PIN photo-detector. The BER and Q-factor values for four atmospheric conditions are 
evaluated. The maximum link range for $16 \times 5$ Gbps system using APD photo-detector is $453 \mathrm{~km}$ achieved for PIN photo-detector it is up to $388 \mathrm{~km}$. In 16x10Gbps system using APD photo-detector maximum link range $370 \mathrm{~km}$ and using PIN photo-detector it is up to $590 \mathrm{~km}$. So it is conclude that $16 x 5$ Gbps system using APD photo-detector having best performance at higher attenuation. For lower attenuation 16x10Gbps system using PIN photo-detector is best.

\section{REFERENCES}

[1] M.Shunmuga , Lakshmi, P.Kanna, "A literature survey on Performance of Free Space Optical communication link under strong trubulence." IOSR Journal of Electronics and Communication Engineering. Volume 3, Issue 5 (Sep-Oct. 2012).

[2] Harneet Kaur and Himali Sarangal, "Simulative Investigation of FSO System using 4X4 Transmitter Receiver Combination Integrated with Various Types of Amplifiers under Different Weather Conditions" International Journal of Signal Processing, Image Processing and Pattern Recognition Vol.9, No.3 (2016), pp.11-16 doi.10.14257/ijsip.2016.9.3.02.(IJSIP journal)

[3] Arun K. Majumdar , “Advanced Free Space Optics (FSO). A Systems Approach”, Volume 186. ISSN 1556-1534 (electronic), ISBN 978-1-4939-0918-6 (eBook), 2015, Springer.
[4]. Mazin Ali, A. Ali, " Performance analysis of WDM-FSO link under turbulence channel ." World Scientific News, volume WSN 56 (2016) 33-44, Issue EISSN 2392-2192 , (2016).

[5] Mazin Ali, A. Ali, "Performance analysis of terrestrial WDMDSO link under different weather condition.” World Scientific News, volume WSN 50 (2016) 160-173, issue EISSN 2392-2192, (2016)

[6] Ashish kumar, Aakash Dhiman, Devender kumar, "Free Space optical communication system under different weather conditions.” IOSR Journal of Engineering ,(e-ISSN: 22503021, p-ISSN: 2278-8719 Vol. 3, Issue 12 (December. 2013), (IOSRJEN)

[7] Aditi and Preeti, "An effort to design a power efficient, long reach WDM-FSO system” ” in Proc. IEEE, 2014 International Conference on Signal Propagation and Computer Technology (ICSPCT), 978-1-4799-3140-8/14/ (C2014 IEEE.

[8] Sooraj Prakesh, Anurag Sharma, "Performance investigation of 40 GB/s DWDM over Free Space Optical communication system using RZ modulation format.” Hindawi Publishing Corporation Advances in Optical Technologies Volume 2016, Article ID 4217302, 8 pages doi.10.1155/2016/4217302 .

[9] Zabih Ghassemlooy, Joaquin Perez \& Erich Leitgeb "On the Performance of FSO Communications Links under Sandstorm Conditions "12th International Conference on Telecommunications - ConTEL 2013, June 26-28, 2013, Zagreb, Croatia. ISBN: 978-953-184-180-1. (2013). 